\title{
Study of Left Ventricular Function in Patients with Acute Myocardial Infarction and Neutrophilia
}

\author{
Ahmed Kamal Matawaa', Mohsen Ali Salama ${ }^{1}$, Mamdouh Attia Mohamed ${ }^{2}$, Abd El-Aziz Rizk Hasan', \\ Mohamad Fawzy Abo-El-Naga Abd El-Rahman ${ }^{1}$ \\ ${ }^{1}$ Department of Cardiology, ${ }^{2}$ Department of Clinical Pathology, Faculty of Medicine Al-Azhar \\ University, Cairo, Egypt \\ *Corresponding author: Mohamad Fawzy Abo-El-Naga Abd El-Rahman, E-mail: \\ dr.m.fawzy2004@gmail.com, Mobile: (+20) 1002059419
}

\begin{abstract}
Background: Acute myocardial infarction is frequently associated with leukocytosis and elevated neutrophil count. Neutrophilia may occur in response to myocardial necrosis which is a potent acute phase stimulus that is associated with a local and systemic inflammatory response.
\end{abstract}

Objective: The aim of this study was to evaluate the left ventricular systolic function in patients with STsegment elevated myocardial infarction and neutrophilia.

Patients and Methods: This prospective study included fifty patients with acute ST-segment elevated myocardial infarction at Cardiology Department, Al-Azhar university Hospitals within the period between January 2016 and June 2018. The patients were divided into two groups: Group (A): Thirty patients with neutrophilia. Group (B): twenty patients without neutrophilia.

Results: Patient's ECG in $1^{\text {st }}$ period showed that all patients had positive ECG in both groups while at $2^{\text {nd }}$ and $3^{\text {rd }}$ periods all patients had negative ECG in both groups with statistically significant differences between $1^{\text {st }}$ period and other period $\left(2^{\text {nd }}\right.$ and $3^{\text {rd }}$ period) where $\mathrm{P}<0.001$ at all. According to neutrophil count there was statistically significant difference between the studied groups in all period where $\mathrm{P}<0.001$ at all period. There was statistically significant difference between the studied groups where $\mathrm{P}<0.001$ at baseline and after 48 hours as regard to EF and FS. There was statistically significant difference between the studied groups according to patients RWMA where $\mathrm{P}<0.001$ at baseline and $\mathrm{P}=0.002$ after 48 hours.

Conclusion: Patients with STEMI associated with neutrophilia are significantly associated with early development of congestive heart failure.

Keywords: Left ventricular function, Myocardial infarction, Neutrophilia

\section{INTRODUCTION}

The relationship between inflammation and myocardial infarction (MI) was suggested more than 50 years ago. Since then, overwhelming evidences supporting this relationship have been obtained from various basic sciences, epidemiological and clinical studies ${ }^{(\mathbf{1})}$.

Determining peripheral leukocyte count is an inexpensive and widely available way to assess the presence of any inflammation. According to the literature, $\mathrm{MI}$ is usually accompanied with peripheral leukocytosis and the leukocytosis is associated with higher rates of short-term mortality and heart failure after myocardial infarction ${ }^{(2)}$.

Neutrophilia may occur in response to myocardial necrosis which is a potent acute phase stimulus that is associated with a local and systemic inflammatory response ${ }^{(3)}$. Also, there are some reports regarding the value of monocyte count in predicting heart failure following $\mathrm{MI}^{(\mathbf{4})}$.

The potential role of neutrophil in promoting coagulation, increasing microvascular permeability and mediating ischemia-reperfusion injury in acute coronary syndrome has been previously prescribed. It was found that relative neutrophilia in patients with ST-elevated myocardial infarction is significantly associated with early development of congestive heart failure and impaired epicardial flow ${ }^{(5)}$.

Leukocytosis, predominantly neutrophilia, has previously been described following ST elevation myocardial infarction (STEMI). The exact contribution of this phenomenon to the clinical outcome of STEMI is yet to be shown ${ }^{(6)}$. High neutrophil counts have been related to angiographically determine coronary atherosclerosis and a poor clinical prognosis ${ }^{(7)}$.

The aim of this study was to evaluate the left ventricular systolic function in patients with STsegment elevated myocardial infarction and neutrophilia.

\section{PATIENTS AND METHODS}


This prospective study included a total of fifty patients with acute ST-segment elevated myocardial infarction, attending at Cardiology Department, Al-Azhar University Hospitals. This study was conducted between January 2016 and June 2018.

Ethical approval:

The study was approved by the Ethics Board of Al-Azhar University and an informed written consent was taken from each participant in the study.

The included subjects were divided into two groups; Group (A) consisted of thirty patients with neutrophilia, Group (B) consisted of twenty patients without neutrophilia.

\section{Inclusion criteria:}

Patients presented with ST-segment elevation acute myocardial infarction (with and without neutrophilia) defined as typical chest pain and either ST-segment elevation in two contiguous leads $\geq 2 \mathrm{~mm}$ in males and $\geq 1.5 \mathrm{~mm}$ in females in V2-3 and / or $\geq 1 \mathrm{~mm}$ in other leads or the new onset of left bundle-branch block with 2-fold elevation of creatine kinase (CK) and creatine kinase-MB (CK-MB) fraction who were eligible for primary percutaneous coronary intervention (PCI).

\section{Exclusions criteria:}

1- Patients with obvious cause of leukocytosis or neutrophilia (e.g. infection). Neutrophilia count is defined as an increase in circulating neutrophils in the peripheral blood. The absolute neutrophil count defines neutrophilia. ANC is found by multiplying the percentage of bands and neutrophils on a differential by the total white blood cell count. Neutrophilia is defined as an elevated circulating neutrophil count $\left(>7.7 \times 10^{\wedge} 9 / \mathrm{L}\right)$ in adults with a normal total WBC count of $<11 \times 10^{\wedge} 9 / \mathrm{L}$. An elevated total WBC count $\left(>11 \times 10^{\wedge} 9 / \mathrm{L}\right)$ and an absolute neutrophil count $>7.7 \times 10^{\wedge} 9 / \mathrm{L}$ is defined as a neutrophilic leukocytosis ${ }^{\left({ }^{(8)}\right.}$.

2- Patients with previous history of myocardial infarction.

\section{All patients were subjected to the following:}

1- Full history taking including age, gender, risk factors for coronary artery disease such as diabetes mellitus, hypertension, smoking, dyslipidemia, family history of premature coronary artery disease and drug history of streptokinase.
- Hypertension was defined as blood pressure of $\geq 140 / 90 \mathrm{mmHg}$ recorded at least two times or current antihypertensive therapy ${ }^{(9)}$.

- Diabetes was defined as fasting plasma glucose of $>126 \mathrm{mg} / \mathrm{dL}$ for at least two measurements or current glucose lowering treatment as defined by the World Health Organization ${ }^{(\mathbf{1 0})}$.

- Hyperlipidemia was defined as total cholesterol of $>200 \mathrm{mg} / \mathrm{dL}$ or a history of elevated serum total cholesterol during the previous 6 months resulting in prescription of a lipid lowering agent ${ }^{(\mathbf{1 1})}$.

2- Thorough physical examination with special emphasis on:

A) Signs of heart failure with estimation of Killip classification degree as follow:

I) No signs of heart failure.

II) Mild heart failure: rales at bases, S3 gallop or elevated JVP.

III) Heart failure: acute pulmonary edema.

IV) Cardiogenic shock (SBP $<90$ $\mathrm{mmHg}$, cyanosis, oliguria, sweating).

B) Mechanical complications such as ventricular septal rupture, acute mitral insufficiency and myocardial free wall rupture causing cardiac tamponade.

3- 12-Lead ECG documented either ST-segment elevation of $>1 \mathrm{~mm}$ in 2 consecutive leads or new onset left bundle-branch block.

4- Complete blood cell count (CBC) was performed for all patients within 12-24 hours of onset of symptoms. After sampling, total WBC and neutrophil counts, were done using CBC H1 machine.

5- Conventional Transthoracic Echocardiography: was done within 48 hours of hospital admission in both groups (mainly to compare the EF \& FS in both groups) and the extent of wall motion abnormalities.

$$
\text { Two dimensional }
$$

echocardiography and Doppler examination was performed using a $1.7-4 \mathrm{MHz}$ transducer (GE Vivid 9 Ultrasound Machine) with simultaneous ECG recording at the laboratory of echocardiography of the cardiology department, Al-Azhar University hospitals by the same observer. All examinations were performed with the patients in the standard left lateral position.

All patients underwent a detailed echocardiography, which included: 
1-Left ventricular measurements were taken using 2-D guided M-mode:

- Left ventricular end diastolic diameter (LVEDd)

- Left ventricular end systolic diameter. (LVESd).

- Inter-ventricular septum thickness (IVST)

- Posterior wall thickness (LVPWT).

- Interventricular septum and posterior wall thickness were measured at end diastole and end systole.

- Assessment of LV systolic function (fraction shortening FS, and LV ejection fraction $\mathrm{EF}$ ).

2- Two Dimensional- Echocardiography: (Apical 4 chamber view, Apical 2 chamber view, long parasternal view) to assess:

- Wall motion abnormalities.

- Assessment of LV functions by Simpson method.

The LV was divided into 16 segments as recommended by American Society of Echocardiography as follows: basal-, midventricului and apical levels of the septum, lateral, anterior and inferior walls and basal- and midventricular levels of the anteroseptal and posterior walls. Regional wall motion was visually assessed considering both endocardial motion and systolic wall thickening.

\section{Statistical analysis:}

Data were fed to the computer and analyzed using IBM SPSS software package version 20.0. (Armonk, NY: IBM Corp) Qualitative data were described using number and percent. The Kolmogorov-Smirnov test was used to verify the normality of distribution Quantitative data were described using range (minimum and maximum), mean, standard deviation and median. Significance of the obtained results was judged at the 5\% level. The used tests were

1 - Chi-square test: For categorical variables, to compare between different groups. For normally distributed quantitative variables, to compare between two studied groups

2 - F-test (ANOVA): For normally distributed quantitative variables, to compare between more than two groups, and Post Hoc test (Tukey) for pairwise comparisons

3 - Kruskal Wallis test: For abnormally distributed quantitative variables, to compare between more than two studied groups, and Post Hoc (Dunn's multiple comparisons test) for

4 - Receiver operating characteristic curve (ROC): It is generated by plotting sensitivity (TP) on $\mathrm{Y}$ axis versus 1-specificity ( $\mathrm{FP}$ ) on $\mathrm{X}$ axis at different cut off values. The area under the ROC curve denotes the diagnostic performance of the test. Area more than 50\% gives acceptable performance and area about $100 \%$ is the best performance for the test. The ROC curve allows also a comparison of performance between two tests.

5 -Sensitivity: The capacity of the test to correctly identify diseased individuals in a population "TRUE POSITIVES". The greater the sensitivity, the smaller the number of unidentified case "false negatives"

6-Specificity: The capacity of the test to correctly exclude individuals who are free of the disease "TRUE NEGATIVES". The greater the specificity, the fewer "false positives" will be included

7 - Positive Predictive value (PPV): The probability of the disease being present, among those with positive diagnostic test results

8 - Negative Predictive value (NPV): The probability that the disease was absent, among those whose diagnostic test results were negative.

\section{RESULTS}

Patient's sex in High neutrophil group showed that $27(90 \%)$ were male and 3(10\%) were female while in Normal neutrophil group $18(90 \%)$ were male and $2(10 \%)$ were female. There was no statistically significant difference between the studied groups where $\mathrm{P}=1.000$. (Table (1).

Patient's age in High neutrophil group ranged between 46.0 - 79.0 years with mean value $62.63 \pm 8.03$ years while in Normal neutrophil group it ranged between $43.0-75.0$ years with mean value $63.45 \pm 7.86$ years. There was no statistically significant difference between the studied groups where $\mathrm{P}=0.724$. Table (1). 


\begin{tabular}{|c|c|c|c|c|c|c|c|c|}
\hline & \multicolumn{2}{|c|}{$\begin{array}{l}\text { Total } \\
(n=50)\end{array}$} & \multicolumn{2}{|c|}{$\begin{array}{l}\text { High Neutrophil } \\
(n=30)\end{array}$} & \multicolumn{2}{|c|}{$\begin{array}{l}\text { Normal } \\
\text { Neutrophil } \\
(\mathbf{n = 2 0})\end{array}$} & \multirow[t]{2}{*}{$\begin{array}{l}\text { Test of } \\
\text { sig. }\end{array}$} & \multirow[t]{2}{*}{$\mathbf{p}$} \\
\hline & \begin{tabular}{|l} 
No. \\
\end{tabular} & $\%$ & No. & $\%$ & No. & $\%$ & & \\
\hline $\begin{array}{l}\text { Sex } \\
\quad \text { Male } \\
\quad \text { Female } \\
\end{array}$ & $\begin{array}{l}45 \\
5 \\
\end{array}$ & $\begin{array}{l}90.0 \\
10.0 \\
\end{array}$ & $\begin{array}{l}27 \\
3 \\
\end{array}$ & $\begin{array}{l}90.0 \\
10.0 \\
\end{array}$ & $\begin{array}{l}18 \\
2 \\
\end{array}$ & $\begin{array}{l}90.0 \\
10.0 \\
\end{array}$ & $\begin{array}{l}\chi^{2}= \\
0.0\end{array}$ & $\begin{array}{l}{ }^{F E} \mathrm{p}= \\
1.000\end{array}$ \\
\hline $\begin{array}{l}\text { Age } \\
\text { Min. - Max. } \\
\text { Mean } \pm \text { SD. } \\
\text { Median } \\
\end{array}$ & \multicolumn{2}{|c|}{$\begin{array}{l}43.0-79.0 \\
62.96 \pm 7.89 \\
64.0\end{array}$} & \multicolumn{2}{|c|}{$\begin{array}{l}46.0-79.0 \\
62.63 \pm 8.03 \\
63.50\end{array}$} & \multicolumn{2}{|c|}{$\begin{array}{l}43.0-75.0 \\
63.45 \pm 7.86 \\
65.0\end{array}$} & $\begin{array}{l}\mathrm{t}= \\
0.355\end{array}$ & 0.724 \\
\hline
\end{tabular}

$\chi^{2}, p: \chi^{2}$ and $p$ values for Chi square test for comparing between the two groups

${ }_{\mathrm{FE}} \mathrm{p}$ : $\mathrm{p}$ value for Fisher Exact for Chi square test for comparing between the two groups

$t, p$ : $t$ and $p$ values for Student $t$-test for comparing between the two groups

Patient's ECG in $1^{\text {st }}$ period showed that all patients had positive ECG in both groups while at $2^{\text {nd }}$ period and $3^{\text {rd }}$ period all patients had negative ECG in both groups with statistically significant differences between $1^{\text {st }}$ period and other period $\left(2^{\text {nd }}\right.$ and $3^{\text {rd }}$ period) where $\mathrm{P}<0.001$ at all. Table (2).

Table (2): Comparison between the two studied groups according to ECG

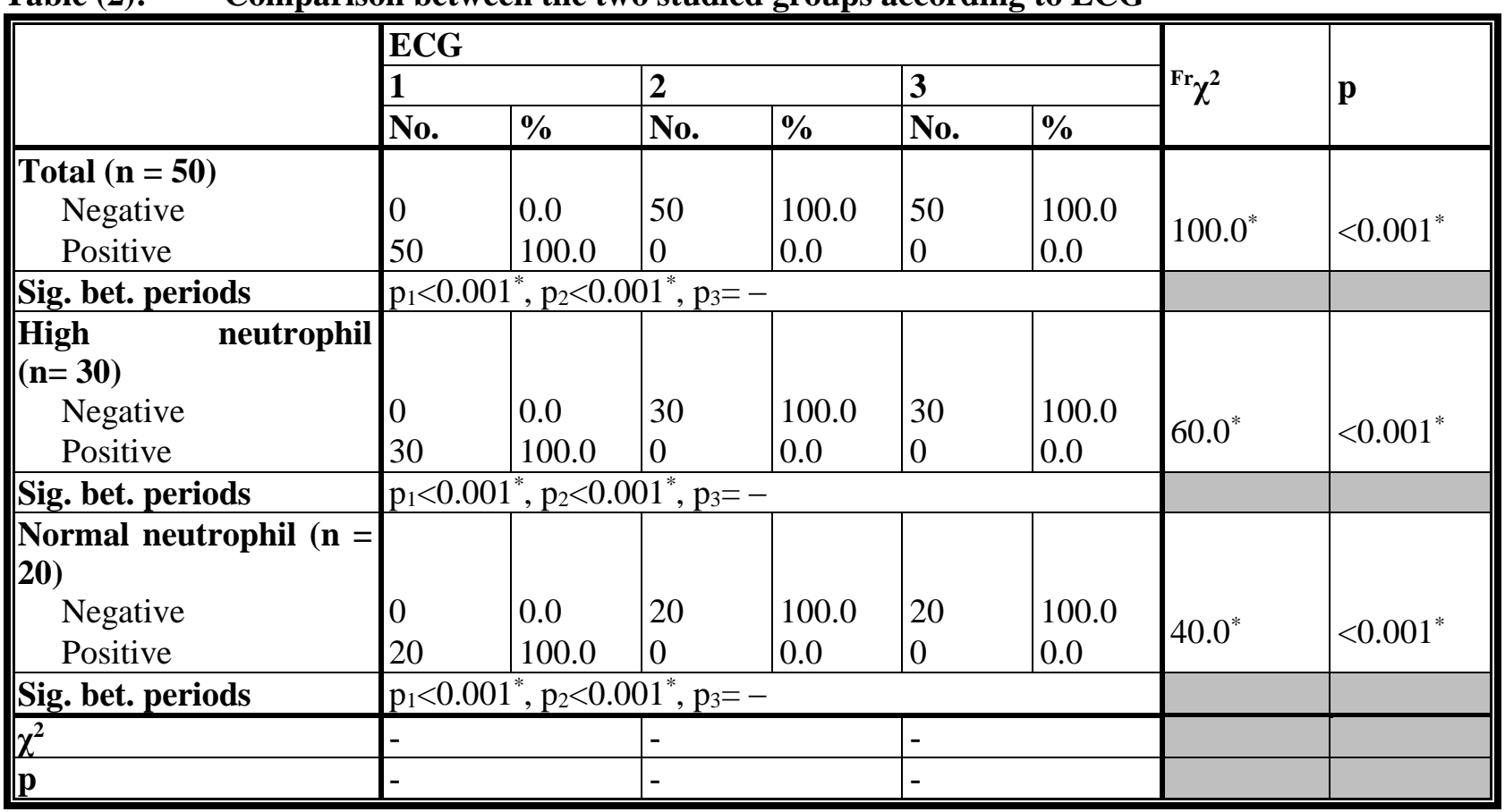

$\chi^{2}$ : Chi square for Friedman test, Sig. bet. periods was done using McNemar test

*: Statistically significant at $\mathrm{p} \leq 0.05$

Patient's WBCs in High neutrophil group in $1^{\text {st }}$ period was ranged between $3.50-17.0$ with mean value $13.60 \pm 2.67$, in $2^{\text {nd }}$ period it was ranged between $4.0-16.0$ with mean value 12.65 \pm 2.42 and at $3^{\text {rd }}$ period it was ranged between 4.0 - 15.0 with mean value $11.90 \pm 2.31$ with statistically significant differences between $1^{\text {st }}$ period and other period ( $2^{\text {nd }}$ and $3^{\text {rd }}$ period $)$ where $\mathrm{P}=0.002$ and $<0.001$ respectively while in Normal neutrophil group in $1^{\text {st }}$ period it was ranged between $4.0-9.0$ with mean value $6.90 \pm 1.77$, in $2^{\text {nd }}$ period it was ranged between $4.50-8.0$ with mean value $6.65 \pm 1.05$ and at $3^{\text {rd }}$ period it was ranged between $4.0-7.0$ with mean value $5.78 \pm$ 1.01 with statistically significant differences between $1^{\text {st }}$ period and $3^{\text {rd }}$ period where $\mathrm{P}<0.001$ and between $2^{\text {nd }}$ period and $3^{\text {rd }}$ period where $\mathrm{P}=0.001$. There was statistically significant difference between the studied groups in all period where $\mathrm{P}<0.001$ at all period. Table (3). 


\begin{tabular}{|c|c|c|c|c|c|}
\hline WBCs $\times 10^{\wedge} 9 / \mathrm{L}$ & 1 & 2 & 3 & $\overline{F r} \chi^{2}$ & $\bar{p}$ \\
\hline \begin{tabular}{|} 
Total $(\mathbf{n}=\mathbf{5 0})$ \\
Min. - Max \\
Mean \pm SD \\
Median
\end{tabular} & $\begin{array}{l}3.50-17.0 \\
10.92 \pm 4.05 \\
12.0\end{array}$ & $\begin{array}{l}4.0-16.0 \\
10.25 \pm 3.56 \\
11.50\end{array}$ & $\begin{array}{l}4.0-15.0 \\
9.45 \pm 3.57 \\
10.75\end{array}$ & $38.426^{*}$ & $<0.001^{*}$ \\
\hline Sig. bet. periods & \multicolumn{3}{|c|}{$\mathrm{p}_{1}=0.007^{*}, \mathrm{p}_{2}<0.001^{*}, \mathrm{p}_{3}=0.001^{*}$} & & \\
\hline \begin{tabular}{|l} 
High neutrophil \\
$(\mathbf{n}=\mathbf{3 0})$ \\
Min. - Max. \\
Mean \pm SD. \\
Median
\end{tabular} & $\begin{array}{l}3.50-17.0 \\
13.60 \pm 2.67 \\
14.0\end{array}$ & $\begin{array}{l}4.0-16.0 \\
12.65 \pm 2.42 \\
13.0\end{array}$ & $\begin{array}{l}4.0-15.0 \\
11.90 \pm 2.31 \\
12.0\end{array}$ & $25.532^{*}$ & $<0.001^{*}$ \\
\hline Sig. bet. periods & \multicolumn{3}{|c|}{$\mathrm{p}_{1}=0.002^{*}, \mathrm{p}_{2}<0.001^{*}, \mathrm{p}_{3}=0.107$} & & \\
\hline \begin{tabular}{|l} 
Normal neutrophil \\
$(\mathbf{n}=\mathbf{2 0})$ \\
Min. - Max. \\
Mean \pm SD. \\
Median
\end{tabular} & $\begin{array}{l}4.0-9.0 \\
6.90 \pm 1.77 \\
7.50\end{array}$ & $\begin{array}{l}4.50-8.0 \\
6.65 \pm 1.05 \\
7.0\end{array}$ & $\begin{array}{l}4.0-7.0 \\
5.78 \pm 1.01 \\
6.0\end{array}$ & $16.545^{*}$ & $<0.001^{*}$ \\
\hline Sig. bet. periods & \multicolumn{3}{|c|}{$\mathrm{p}_{1}=0.693, \mathrm{p}_{2}<0.001^{*}, \mathrm{p}_{3}=0.001^{*}$} & & \\
\hline
\end{tabular}

$\chi^{2}$ : Chi square for Friedman test, Sig. bet. Periods was done using Post Hoc Test (Dunn-Bonferroni)

$*$ : Statistically significant at $\mathrm{p} \leq 0.05$

Patient's HB in High neutrophil group in $1^{\text {st }}$ period was ranged between $10.0-15.0$ with mean value $12.78 \pm 1.54$, in $2^{\text {nd }}$ period it was ranged between $10.0-14.0$ with mean value $11.95 \pm 1.29$ and at $3^{\text {rd }}$ period it was ranged between $1.50-13.50$ with mean value $11.37 \pm 2.16$ with statistically significant differences between $1^{\text {st }}$ period and other period $\left(2^{\text {nd }}\right.$ and $3^{\text {rd }}$ period) where $\mathrm{P}<0.001$ and $<0.001$ respectively while in Normal neutrophil group in $1^{\text {st }}$ period it was ranged between $10.50-$
15.0 with mean value $12.48 \pm 1.25$, in $2^{\text {nd }}$ period it was ranged between 10.0 - 14.0 with mean value $11.88 \pm 1.12$ and at $3^{\text {rd }}$ period it was ranged between $10.0-13.0$ with mean value $11.43 \pm 0.95$ with statistically significant differences between $1^{\text {st }}$ period and other period ( $2^{\text {nd }}$ and $3^{\text {rd }}$ period $)$ where $\mathrm{P}<0.001$ and $<0.001$ respectively and between $2^{\text {nd }}$ period and $3^{\text {rd }}$ period where $\mathrm{P}<0.001$. There was no statistically significant difference between the studied groups in all period. Table (4).

Table (4): Comparison between the two studied groups according to HB

\begin{tabular}{|c|c|c|c|c|c|}
\hline HB g/dl & 1 & 2 & 3 & $\mathbf{F}$ & $\mathbf{p}$ \\
\hline $\begin{array}{c}\text { Total }(\mathbf{n}=\mathbf{5 0}) \\
\text { Min. }- \text { Max. } \\
\text { Mean } \pm \text { SD } \\
\text { Median }\end{array}$ & $\begin{array}{l}10.0-15.0 \\
12.66 \pm 1.42 \\
12.50\end{array}$ & $\begin{array}{l}10.0-14.0 \\
11.92 \pm 1.21 \\
12.0\end{array}$ & $\begin{array}{l}1.50-13.50 \\
11.39 \pm 1.76 \\
11.50\end{array}$ & $29.037^{*}$ & $<0.001^{*}$ \\
\hline Sig. bet. periods & \multicolumn{3}{|c|}{$\mathrm{p}_{1}<0.001^{*}, \mathrm{p}_{2}<0.001^{*}, \mathrm{p}_{3}=0.007^{*}$} & & \\
\hline $\begin{array}{l}\text { High neutrophil } \\
(\mathbf{n}=\mathbf{3 0}) \\
\text { Min. }- \text { Max. } \\
\text { Mean } \pm \text { SD } \\
\text { Median }\end{array}$ & $\begin{array}{l}10.0-15.0 \\
12.78 \pm 1.54 \\
13.0\end{array}$ & $\begin{array}{l}10.0-14.0 \\
11.95 \pm 1.29 \\
12.0\end{array}$ & $\begin{array}{l}1.50-13.50 \\
11.37 \pm 2.16 \\
11.50\end{array}$ & $13.619^{*}$ & $0.001^{*}$ \\
\hline Sig. bet. periods & \multicolumn{3}{|c|}{$\mathrm{p}_{1}<0.001^{*}, \mathrm{p}_{2}<0.001^{*}, \mathrm{p}_{3}=0.072$} & & \\
\hline $\begin{array}{l}\text { Normal neutrophil } \\
(\mathbf{n}=\mathbf{2 0}) \\
\text { Min. }- \text { Max. } \\
\text { Mean } \pm \text { SD. } \\
\text { Median }\end{array}$ & $\begin{array}{l}10.50-15.0 \\
12.48 \pm 1.25 \\
12.25\end{array}$ & $\begin{array}{l}10.0-14.0 \\
11.88 \pm 1.12 \\
11.75\end{array}$ & $\begin{array}{l}10.0-13.0 \\
11.43 \pm 0.95 \\
11.25\end{array}$ & $68.772^{*}$ & $<0.001^{*}$ \\
\hline Sig. bet. periods & \multicolumn{3}{|c|}{$\mathrm{p}_{1}<0.001^{*}, \mathrm{p}_{2}<0.001^{*}, \mathrm{p}_{3}<0.001^{*}$} & & \\
\hline
\end{tabular}

F, p: $F$ and $p$ values for F test (ANOVA) with repeated measures, Sig. bet. Periods was done using Post Hoc Test (LSD)

*: Statistically significant at $\mathrm{p} \leq 0.05$

Patient's platelets in High neutrophil group in $1^{\text {st }}$ period was ranged between $180.0-422.0$ with mean value $294.2 \pm 67.79$, in $2^{\text {nd }}$ period it was ranged between $175.0-400.0$ with mean value
$284.4 \pm 66.26$ and at $3^{\text {rd }}$ period it was ranged between $170.0-390.0$ with mean value $280.0 \pm$ 66.54 with statistically significant differences between $1^{\text {st }}$ period and other period $\left(2^{\text {nd }}\right.$ and $3^{\text {rd }}$ 
period) where $\mathrm{P}<0.001$ and $<0.001$ respectively while in Normal neutrophil group in $1^{\text {st }}$ period it was ranged between 170.0 - 420.0 with mean value $269.80 \pm 66.95$, in $2^{\text {nd }}$ period it was ranged between $165.0-400.0$ with mean value $259.8 \pm 63.13$ and at $3^{\text {rd }}$ period it was ranged between $153.0-384.0$ with mean value $250.4 \pm 59.61$ with statistically significant differences between $1^{\text {st }}$ period and other period $\left(2^{\text {nd }}\right.$ and $3^{\text {rd }}$ period) where $\mathrm{P}<0.001$ and $<0.001$ respectively and between $2^{\text {nd }}$ period and $3^{\text {rd }}$ period where $\mathrm{P}<0.001$. There was no statistically significant difference between the studied groups in all period. Table (5).

Table (5): Comparison between the two studied groups according to platelets

\begin{tabular}{|c|c|c|c|c|c|}
\hline Platelets $\times 10^{\wedge} 9 / \mathrm{L}$ & 1 & 2 & 3 & $\mathbf{F}$ & $\mathbf{p}$ \\
\hline \begin{tabular}{|l} 
Total $(\mathbf{n}=\mathbf{5 0})$ \\
Min. - Max. \\
Mean \pm SD \\
Median
\end{tabular} & $\begin{array}{l}170.0-422.0 \\
284.4 \pm 67.85 \\
278.5\end{array}$ & $\begin{array}{l}165.0-400.0 \\
274.6 \pm 65.52 \\
270.0\end{array}$ & $\begin{array}{l}153.0-390.0 \\
268.1 \pm 64.90 \\
260.0\end{array}$ & $36.224^{*}$ & $<0.001^{*}$ \\
\hline Sig. bet. periods & \multicolumn{3}{|c|}{$\mathrm{p}_{1}<0.001^{*}, \mathrm{p}_{2}<0.001^{*}, \mathrm{p}_{3}=0.005^{*}$} & & \\
\hline $\begin{array}{l}\text { High neutrophil } \\
(\mathbf{n}=\mathbf{3 0}) \\
\text { Min. }- \text { Max. } \\
\text { Mean } \pm \text { SD } \\
\text { Median }\end{array}$ & $\begin{array}{l}180.0-422.0 \\
294.2 \pm 67.79 \\
293.5\end{array}$ & $\begin{array}{l}175.0-400.0 \\
284.4 \pm 66.26 \\
278.5\end{array}$ & $\begin{array}{l}170.0-390.0 \\
280.0 \pm 66.54 \\
265.0\end{array}$ & $11.927^{*}$ & $<0.001^{*}$ \\
\hline Sig. bet. periods & \multicolumn{3}{|c|}{$\mathrm{p}_{1}<0.001^{*}, \mathrm{p}_{2}<0.001^{*}, \mathrm{p}_{3}=0.211$} & & \\
\hline $\begin{array}{l}\text { Normal neutrophil } \\
(\mathbf{n}=\mathbf{2 0}) \\
\text { Min. }- \text { Max. } \\
\text { Mean } \pm \text { SD. } \\
\text { Median }\end{array}$ & $\begin{array}{l}170.0-420.0 \\
269.80 \pm 66.95 \\
266.0\end{array}$ & $\begin{array}{l}165.0-400.0 \\
259.8 \pm 63.13 \\
255.0\end{array}$ & $\begin{array}{l}153.0-384.0 \\
250.4 \pm 59.61 \\
247.5\end{array}$ & $60.247^{*}$ & $<0.001^{*}$ \\
\hline Sig. bet. periods & \multicolumn{3}{|c|}{$\mathrm{p}_{1}<0.001^{*}, \mathrm{p}_{2}<0.001^{*}, \mathrm{p}_{3}<0.001^{*}$} & & \\
\hline
\end{tabular}

F, p: F and p values for F test (ANOVA) with repeated measures, Sig. bet. Periods was done using Post Hoc Test (LSD)

*: Statistically significant at $\mathrm{p} \leq 0.05$

Patient's Neutrophil in High neutrophil group in $1^{\text {st }}$ period was ranged between $55.0-95.0$ with mean value $87.40 \pm 8.59$, in $2^{\text {nd }}$ period it was ranged between 54.0 - 93.0 with mean value 85.50 \pm 8.38 and at $3^{\text {rd }}$ period it was ranged between 52.0 - 92.0 with mean value $83.33 \pm 8.35$ with statistically significant differences between $1^{\text {st }}$ period and other period $\left(2^{\text {nd }}\right.$ and $3^{\text {rd }}$ period $)$ where $\mathrm{P}<0.001$ and $<0.001$ respectively and between $2^{\text {nd }}$ period and $3^{\text {rd }}$ period where $\mathrm{P}<0.001$ while in Normal neutrophil group in $1^{\text {st }}$ period it was ranged between $47.0-79.0$ with mean value $62.75 \pm$ 10.49 , in $2^{\text {nd }}$ period it was ranged between $45.0-$ 78.0 with mean value $60.60 \pm 10.53$ and at $3^{\text {rd }}$ period it was ranged between $42.0-75.0$ with mean value $57.30 \pm 10.27$ with statistically significant differences between $1^{\text {st }}$ period and other period $\left(2^{\text {nd }}\right.$ and $3^{\text {rd }}$ period $)$ where $\mathrm{P}<0.001$ and $<0.001$ respectively and between $2^{\text {nd }}$ period and $3^{\text {rd }}$ period where $\mathrm{P}=0.002$. There was statistically significant difference between the studied groups in all period where $\mathrm{P}<0.001$ at all period. Table (6).

Table (6): Comparison between the two studied groups according to neutrophil

\begin{tabular}{||l|l|l|l|l|l||}
\hline Neutrophil \% & $\mathbf{1}$ & $\mathbf{2}$ & $\mathbf{3}$ & ${ }^{\mathrm{Fr}} \boldsymbol{\chi}^{2}$ & $\mathbf{p}$ \\
\hline Total (n= 50) & & & & & \\
Min. - Max. & $47.0-95.0$ & $45.0-93.0$ & $42.0-92.0$ & & \\
Mean \pm SD. & $77.54 \pm 15.33$ & $75.54 \pm 15.37$ & $72.92 \pm 15.75$ & $100.0^{*}$ & $<0.001^{*}$ \\
Median & 85.0 & 84.0 & 81.50 & & \\
\hline
\end{tabular}




\begin{tabular}{|c|c|c|c|c|c|}
\hline Sig. bet. periods & \multicolumn{3}{|c|}{$\mathrm{p}_{1}<0.001^{*}, \mathrm{p}_{2}<0.001^{*}, \mathrm{p}_{3}<0.001^{*}$} & \multirow[b]{2}{*}{$60.0^{*}$} & \multirow[b]{2}{*}{$<0.001^{*}$} \\
\hline $\begin{array}{l}(\mathbf{n}=\mathbf{3 0}) \\
\text { Min. }- \text { Max. } \\
\text { Mean } \pm \text { SD } \\
\text { Median }\end{array}$ & $\begin{array}{l}55.0-95.0 \\
87.40 \pm 8.59 \\
90.0\end{array}$ & $\begin{array}{l}54.0-93.0 \\
85.50 \pm 8.38 \\
88.0\end{array}$ & $\begin{array}{l}52.0-92.0 \\
83.33 \pm 8.35 \\
85.0\end{array}$ & & \\
\hline Sig. bet. periods & \multicolumn{3}{|c|}{$\mathrm{p}_{1}<0.001^{*}, \mathrm{p}_{2}<0.001^{*}, \mathrm{p}_{3}<0.001^{*}$} & & \\
\hline $\begin{array}{l}\text { Normal neutrophil } \\
(\mathbf{n}=\mathbf{2 0}) \\
\text { Min. }- \text { Max. } \\
\text { Mean } \pm \text { SD. } \\
\text { Median }\end{array}$ & $\mid \begin{array}{l}47.0-79.0 \\
62.75 \pm 10.49 \\
60.50\end{array}$ & $\begin{array}{l}45.0-78.0 \\
60.60 \pm 10.53 \\
59.0\end{array}$ & $\begin{array}{l}42.0-75.0 \\
57.30 \pm 10.27 \\
55.0\end{array}$ & $40.0^{*}$ & $<0.001^{*}$ \\
\hline Sig. bet. periods & \multicolumn{3}{|c|}{$\mathrm{p}_{1}=0.002^{*}, \mathrm{p}_{2}<0.001^{*}, \mathrm{p}_{3}=0.002^{*}$} & & \\
\hline
\end{tabular}

$\chi^{2}$ : Chi square for Friedman test, Sig. bet. Periods was done using Post Hoc Test (Dunn-Bonferroni)

*: Statistically significant at $\mathrm{p} \leq 0.05$

Patient's EF\% in cases group at baseline was ranged between $28.0-66.0$ with mean value 45.40 \pm 10.72 and after 48 hours it was ranged between 26.0 - 65.0 with mean value $43.57 \pm 11.02$ with statistically significant differences between baseline and after 48 hours where $\mathrm{P}<0.001$ while control group in baseline was ranged between 29.0 -71.0 with mean value $58.40 \pm 10.72$ and after 48 hours it was ranged between $27.0-68.0$ with mean value $57.40 \pm 10.65$ with statistically significant differences between baseline and after 48 hours where $\mathrm{P}=0.031$. There was statistically significant difference between the studied groups where $\mathrm{P}<0.001$ at baseline and after 48 hours. Table (7).

Patient's FS\% in cases group at baseline was ranged between 14.20 - 33.60 with mean value $23.12 \pm 5.47$ and after 48 hours it was ranged between $2.40-33.10$ with mean value $21.50 \pm 6.68$ with statistically significant differences between baseline and after 48 hours where $\mathrm{P}=0.026$ while control group in baseline was ranged between $14.70-36.60$ with mean value $29.75 \pm 5.50$ and after 48 hours it was ranged between $13.70-34.60$ with mean value $29.23 \pm 5.44$ with statistically significant differences between baseline and after 48 hours where $\mathrm{P}=0.034$. There was statistically significant difference between the studied groups where $\mathrm{P}<0.001$ at baseline and after 48 hours. Table (7).

Patient's RWMI in cases group at baseline was ranged between $1.0-2.90$ with mean value $1.76 \pm 0.56$ and after 48 hours it was ranged between $1.0-2.70$ with mean value $1.63 \pm 0.54$ with statistically significant differences between baseline and after 48 hours where $\mathrm{P}<0.001$ while control group in baseline was ranged between 1.0 - 3.0 with mean value $1.29 \pm 0.49$ and after 48 hours it was ranged between $1.0-2.80$ with mean value $1.23 \pm 0.44$ with statistically significant differences between baseline and after 48 hours where $\mathrm{P}=0.008$. There was statistically significant difference between the studied groups where $\mathrm{P}<0.001$ at baseline and $\mathrm{P}=0.002$ after 48 hours. Table (7). 
Table (7): Comparison between the two studied groups according to ECHO.

\begin{tabular}{|c|c|c|c|c|c|c|}
\hline & ЕСHO & $\begin{array}{l}\text { Total } \\
(n=50)\end{array}$ & $\begin{array}{l}\text { Cases } \\
(\mathbf{n}=\mathbf{3 0})\end{array}$ & $\begin{array}{l}\text { Control } \\
(\mathbf{n}=\mathbf{2 0})\end{array}$ & $\begin{array}{l}\text { Test of } \\
\text { sig. }\end{array}$ & $\mathbf{p}$ \\
\hline \multirow{3}{*}{$\frac{8}{x}$} & $\begin{array}{l}\text { Baseline } \\
\text { Min. - Max. } \\
\text { Mean } \pm \text { SD. } \\
\text { Median }\end{array}$ & $\begin{array}{l}28.0-71.0 \\
50.60 \pm 12.41 \\
52.50\end{array}$ & $\begin{array}{l}28.0-66.0 \\
45.40 \pm 10.72 \\
45.50\end{array}$ & $\begin{array}{l}29.0-71.0 \\
58.40 \pm 10.72 \\
60.0\end{array}$ & $\begin{array}{l}t= \\
4.201^{*}\end{array}$ & $<0.001^{*}$ \\
\hline & $\begin{array}{l}\text { After } 48 \text { hours } \\
\text { Min.-Max. } \\
\text { Mean } \pm \text { SD. } \\
\text { Median }\end{array}$ & $\begin{array}{l}26.0-68.0 \\
49.10 \pm 12.76 \\
50.50\end{array}$ & $\begin{array}{l}26.0-65.0 \\
43.57 \pm 11.02 \\
43.50\end{array}$ & $\begin{array}{l}27.0-68.0 \\
57.40 \pm 10.65 \\
60.0\end{array}$ & $\begin{array}{l}t= \\
4.405^{*}\end{array}$ & $<0.001^{*}$ \\
\hline & ${ }^{t_{1}} \mathbf{p}_{1}$ & $<0.001^{*}$ & $<0.001^{*}$ & $0.031^{*}$ & & \\
\hline \multirow{3}{*}{ 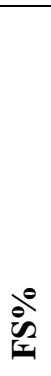 } & $\begin{array}{l}\text { Baseline } \\
\text { Min.-Max. } \\
\text { Mean } \pm \text { SD. } \\
\text { Median }\end{array}$ & $\begin{array}{l}14.20-36.60 \\
25.77 \pm 6.34 \\
26.75\end{array}$ & $\begin{array}{l}14.20-33.60 \\
23.12 \pm 5.47 \\
23.15\end{array}$ & $\begin{array}{l}14.70-36.60 \\
29.75 \pm 5.50 \\
30.60\end{array}$ & $\begin{array}{l}t= \\
4.192^{*}\end{array}$ & $<0.001^{*}$ \\
\hline & $\begin{array}{l}\text { After } 48 \text { hours } \\
\text { Min.Max. } \\
\text { Mean } \pm \text { SD. } \\
\text { Median }\end{array}$ & $\begin{array}{l}2.40-34.60 \\
24.59 \pm 7.25 \\
25.75\end{array}$ & $\begin{array}{l}2.40-33.10 \\
21.50 \pm 6.68 \\
21.65\end{array}$ & $\begin{array}{l}13.70-34.60 \\
29.23 \pm 5.44 \\
30.60\end{array}$ & $\begin{array}{l}t= \\
4.304^{*}\end{array}$ & $<0.001^{*}$ \\
\hline & ${ }^{t} \mathbf{p}_{1}$ & $0.008^{*}$ & $0.026^{*}$ & $0.034^{*}$ & & \\
\hline \multirow{3}{*}{ 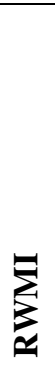 } & $\begin{array}{l}\text { Baseline } \\
\text { Min.-Max. } \\
\text { Mean } \pm \text { SD. } \\
\text { Median }\end{array}$ & $\begin{array}{l}1.0-3.0 \\
1.57 \pm 0.58 \\
1.35\end{array}$ & $\begin{array}{l}1.0-2.90 \\
1.76 \pm 0.56 \\
1.65\end{array}$ & $\begin{array}{l}1.0-3.0 \\
1.29 \pm 0.49 \\
1.10\end{array}$ & $\begin{array}{l}\mathrm{U}= \\
117.0^{*}\end{array}$ & $<0.001^{*}$ \\
\hline & $\begin{array}{l}\text { After } 48 \text { hours } \\
\text { Min.-Max. } \\
\text { Mean } \pm \text { SD. } \\
\text { Median }\end{array}$ & $\begin{array}{l}1.0-2.80 \\
1.47 \pm 0.54 \\
1.20\end{array}$ & $\begin{array}{l}1.0-2.70 \\
1.63 \pm 0.54 \\
1.55\end{array}$ & $\begin{array}{l}1.0-2.80 \\
1.23 \pm 0.44 \\
1.05\end{array}$ & $\begin{array}{l}U= \\
143.50^{*}\end{array}$ & $0.002^{*}$ \\
\hline & $\mathbf{U}_{\mathbf{p}_{1}}$ & $<0.001^{*}$ & $<0.001^{*}$ & $0.008^{*}$ & & \\
\hline
\end{tabular}

$\mathrm{t}, \mathrm{p}$ : $\mathrm{t}$ and $\mathrm{p}$ values for Student t-test for comparing between the two groups

$\mathrm{U}, \mathrm{p}$ : $\mathrm{U}$ and $\mathrm{p}$ values for Mann Whitney test for comparing between the two groups

tp $p_{1}$ p values for $\mathrm{p}$ values for Paired t-test for comparing between baseline and after 48 hours in each group and total sample

${ }^{\mathrm{U}} \mathrm{p}_{1}$ : $\mathrm{p}$ values for $\mathrm{p}$ values for Wilcoxon signed ranks test for comparing between baseline and after 48 hours in each group and total sample

*: Statistically significant at $\mathrm{p} \leq 0.05$

\section{DISCUSSION}

The aim of this study was to evaluate the left ventricular function in patients with ST-segment elevated myocardial infarction and neutrophilia. The study included fifty patients with acute STsegment elevated myocardial infarction at cardiology department, Al-Azhar university hospital within the period between January 2016 and June 2018.

All patients were subjected to clinical history including drug history of streptokinase, clinical examination, and routine lab. Investigations, serial 12- lead ECG and daily CBC till hospital discharge. Echocardiography was done within 48 hours of hospital admission in both groups (mainly to compare the ejection fraction and fraction shortening in both groups) and the extent of wall motion abnormalities. Patients with obvious cause of leukocytosis or neutrophilia were excluded as well as patients with previous history of myocardial infarction.

We performed a single CBC analysis to show the value of this inexpensive and widely available test in risk stratification post-STEMI complications.

Association between higher neutrophil count and heart failure has been stressed in several studies. Chia et al. ${ }^{(12)}$ showed that elevated leukocyte and neutrophil counts after primary PCI in patients with STEMI were associated with larger myocardial infarct size and lower LVEF and were independent predictors of cardiovascular outcome. Similarly, our study patients with evidence of heart failure either as pulmonary edema or cardiogenic shock had higher WBC counts, neutrophils.

Our study found that there was highly significant statistically relation between elevated neutrophil count and myocardial infarction size and 
LV function in patients with acute ST elevated myocardial infarction $(\mathrm{P}<0.001)$ and similar to a study done by Chia $\boldsymbol{e t}$ al. ${ }^{(12)}$ who studied the relation between leukocyte and neutrophil counts with infarct size and the left ventricular ejection fraction (LVEF) after primary PCI. Total leukocyte and neutrophil counts obtained 24 hours after PCI were significantly correlated with infarct size $(\mathrm{p}$ $<0.001$ ) and inversely correlated with the LVEF ( $p$ $<0.001)$. Patients with elevated leukocyte and neutrophil counts had larger infarct sizes $(12.5 \%$ vs $5 \%$ and $13.5 \%$ vs $5 \%$, respectively, $\mathrm{p}<0.001$ ). The highest neutrophil quartile was associated with increased 180-day composite cardiac events (19\% vs $20 \%$ vs $23 \%$ vs $45 \%$, log-rank p <0.001). Elevated leukocyte and neutrophil counts independently predicted adverse cardiac events (hazard ratios 2.5 and 2.2, respectively, $\mathrm{p}=0.001$ ). They concluded that, elevated leukocyte and neutrophil counts in patients with ST-segment elevation myocardial infarctions are directly related to myocardial infarct size and the LVEF and are independent predictors of cardiovascular outcomes.

Same results were obtained by Lorraine $\boldsymbol{e t} \boldsymbol{a l}$. (13) as outcomes measures included clinical episodes of CHF with confirmatory chest roentgenogram findings and/or echocardiographic evidence of contractile dysfunction. CHF developed in $43 \%$ of the cohort. Of these, $92.5 \%$ had relative neutrophilia (neutrophil percentage $>65 \%$ ) compared with $45 \%$ of those in whom CHF did not develop. Multivariable analysis revealed a highly significant association between relative neutrophilia and the subsequent development of CHF (odds ratio 14.3; 95\% confidence interval 5.2 to 39.3). in our study there was a highly significant decrease in LV EF and FS in patients with neutrophilia on admission and after 48 hours measured by echocardiography $(\mathrm{P}<0.001)$

Börekçi et al. ${ }^{(14)}$ had a positive concordant with our study as they concluded that in patients with anterior STEMI, initial NLR and NT-proBNP measured 24 hours after admission may be useful for predicting adverse cardiovascular events including left VR. Echocardiography was performed during admission and at six months after myocardial infarction. VR was defined as at least $20 \%$ increase from baseline in left ventricular enddiastolic volume. They found that Compared with the non-VR group, peak creatine kinase MB (CKMB), NT-proBNP (24 h), neutrophil/lymphocyte ratio, presence of diabetes, no-reflow frequency and wall motion score index were significantly higher in patients with VR ( $\mathrm{p}<0.05$ for all). Peak CK-MB, NT-proBNP (24 h), WMSI and diabetes incidence were associated with VR. in our study we found that there was a highly significant decrease in LV EF and FS in patients with neutrophilia on admission and after 48 hours measured by echocardiography $(\mathrm{P}<0.001)$

Also Klein et al. ${ }^{(15)}$ concluded that high levels of neutrophils and a high NLR upon admission for STEMI were independently associated with an increased risk of developing late cardiogenic shock and, additionally, both biomarkers showed association to 30-day mortality. Patients in the highest quartile of neutrophils (OR:2.54; 95\% CI: 1.40-4.60; $\mathrm{p}=0.002)$ and NLR (OR:3.64; 95\%CI: 2.02-6.54; $\mathrm{p}<0.0001$ ) were at increased risk of developing late cardiogenic shock compared to patients in the lower quartiles, whereas there was no risk difference across quartiles regarding development of early cardiogenic shock. Both biomarkers correlated strongly to an increased 30-day mortality $(\mathrm{P}<0.0001)$ and, moreover, a high level of neutrophils was independently associated with 30-day mortality (HR:1.95; 95\%CI: 1.25-3.03; $\mathrm{p}=0.003)$. In our study we observed that patients with acute ST elevated myocardial infarction who had elevated leukocyte and neutrophil counts had the adverse clinical outcomes $(\mathrm{P}<0.001)$

Oncel et al. (16) had also a positive concordance with our results regarding adverse outcomes. Patients were divided into 3 groups by use of GRACE risk score. The association between NLR and GRACE risk score was assessed. The NLR showed a proportional increase correlated with GRACE risk score $(\mathrm{P}<.001)$. The occurrence of in-hospital cardiac death, re infarction, or newonset heart failure was significantly related to NLR at admission ( $\mathrm{P}<.001)$. Likewise, NLR and GRACE risk score showed a significant positive correlation $(\mathrm{r}=.803, \mathrm{P}<.001)$. They concluded that the NLR is significantly associated with adverse inhospital outcomes, independent of GRACE risk score in patients with STEMI.as we observed that patients with acute ST elevated myocardial infarction who had elevated neutrophil counts had the adverse clinical outcomes $(\mathrm{P}<.001)$.

Tanriverdi et al. ${ }^{(17)}$ had an agreement with our results regarding adverse outcomes in patients with STEMI and elevated neutrophil count in another way as they reported that neutrophil-tolymphocyte ratio is independently associated with the presence of fragmented QRS in acute STEMI patients. Combined use of both parameters provides additional prognostic contribution for identifying patients at higher cardiac risk. Fragmented QRS (fQRS) is a convenient marker of myocardial scar evaluated by 12-lead electrocardiogram (ECG) recording. They found 
that Patients with fQRS had significantly higher NLR, and in-hospital mortality rate compared to patients with no-fQRS. Multivariate analysis showed that NLR was an independent predictor of the presence of fQRS $(\mathrm{P}=0.001)$. When patients were stratified by fQRS and cut-off value of NLR, in-hospital mortality gradually increased $(\mathrm{P}<0.001)$.

Our results were close to that documented by Arbel et al. ${ }^{(18)}$ who studied a group of 538 consecutive STEMI patients, high NLR (NLR $\geq$ $6.5 \%$ ) was independently associated with increased 30-day and 5-years mortality rates (odds ratio, $15.8 ; 95 \%$ confidence interval, $1.6-154 ; \mathrm{P}=0.018$; and hazard ratio, $2.2 ; 95 \%$ confidence interval, 1.04-4.8; $\mathrm{P}=0.039$, respectively). High NLR was also independently associated with lower EF (49 \pm 8 vs $46 \pm 8 ; \mathrm{P}<0.001)$ and fewer hospital complications. They concluded that in patients presenting with STEMI, high NLR was independently associated with lower EF, fewer hospital complications, and higher mortality rates up to 5 years. in our study we found that there was a highly significant decrease in LV EF and FS in patients with neutrophilia on admission and after 48 hours measured by echocardiography ( $\mathrm{P}$ $<0.001)$

Yalcinkaya et al. (19) investigated the relationship between neutrophil-to-lymphocyte ratio and electrocardiographic ischemia grade in STEMI patients. Patients were classified into two groups according to the ischemia grade presented on the admission ECG, as grade 2 ischemia (G2I) and grade 3 ischemia (G3I).They found that Patients with G3I had significantly lower mean left ventricular ejection fraction than those in G2I $(44.58 \pm 7.23$ vs. $48.44 \pm 7.61, \mathrm{p}=0.001)$. As expected, in-hospital mortality rate increased proportionally with the increase in ischemia grade $(p=0.036)$. There were significant differences in percentage of lymphocytes $(\mathrm{p}=0.010)$ and percentage of neutrophils $(\mathrm{p}=0.004)$, and therefore, NLR was significantly different between G2I and G3I patients ( $p<0.001)$. Multivariate logistic regression analysis revealed that only NLR was the independent variable with a significant effect on ECG ischemia grade (odds ratio $=1.254$, 95\% confidence interval 1.120-1.403, $\mathrm{p}<0.001$ ). so they concluded that there is an association between G3I and elevated NLR in patients with STEMI.our study also found that patients with STEMI who had a high neutrophil count had significantly lower mean left ventricular ejection fraction than those without elevated neutrophil count.

Our results was concordant to that demonstrated by Gul $\boldsymbol{e t}$ al. ${ }^{(20)}$ who investigated if neutrophil lymphocyte ratio (NLR) predicts inhospital adverse events and mortality, and short term (30-day) mortality in ST-elevated myocardial infarction (STEMI) patients thrombolysed with streptokinase (SK). Patients in high NLR group had higher rate of complications $(63.5 \%$ vs. $25.5 \%$, p $<0.0001)$ and death $(19.2 \%$ vs. $11.1 \%, \mathrm{p}=0.046)$ in hospital than those in low NLR group. Cardiogenic shock (27.5\% vs. $11.1 \%, \mathrm{p}<0.0001)$, heart failure $(19.2 \%$ vs. $7.2 \%, \mathrm{p}=0.002)$, arrhythmias $(18 \%$ vs. $6.5 \%, \mathrm{p}<0.0001)$, re infarct/angina (9.6\% vs. $2 \%$ $\mathrm{p}=0.004)$ occurred more in high NLR group. Median NLR in patients died was higher than those discharged alive (7.46 vs. 4.70, p <0.0001). Regression analysis showed NLR an independent predictor of mortality (OR 1.131 at $95 \% \mathrm{CI}, \mathrm{p}=$ 0.029). They concluded that a high neutrophil lymphocyte ratio (NLR) predicted increased in hospital complication rate, and in-hospital as well as 30-day mortality in STEMI patients thrombolysed with streptokinase. In our study we observed that patients with acute ST elevated myocardial infarction who had elevated leukocyte and neutrophil counts had the adverse clinical outcomes $(\mathrm{P}<0.001)$.

Kirtane et al. ${ }^{(21)}$ had a positive concordance with our results as they found that absolute and relative neutrophilia were associated with adverse angiographic outcomes. Patients with a closed infarct-related artery at 90 minutes (Thrombolysis In Myocardial Infarction [TIMI] grade 0/1 flow) had higher neutrophil counts $(8.8+/-3.8$ vs $7.6+/-$ $3.0, \mathrm{p}=0.02$ ) but no difference in the percentage of neutrophils than patients with an open artery. Higher neutrophil counts were also mildly correlated with longer corrected TIMI frame counts $(\mathrm{CTFC})$ in the infarct-related artery $(\mathrm{r}=0.14, \mathrm{p}=$ 0.02 ). Patients with impaired myocardial perfusion by TIMI myocardial perfusion grade (TMPG) had a greater percentage of neutrophils $(\mathrm{p}=0.047)$ but no detectable difference in neutrophil counts $(\mathrm{p}=$ 0.24). Higher neutrophil counts remained independently associated with both closed arteries and CTFC in multivariable models controlling for age, left anterior descending artery infarct location, time to treatment, and pulse and blood pressure on admission. They found that a greater percentage of neutrophils remained independently associated with impaired microvascular perfusion in a similar multivariable model.

Same results obtained by Fiarresga $\boldsymbol{e t}$ al. (22), who concluded that in myocardial infarction patients undergoing mechanical revascularization, an intense neutrophil response (routinely, easily and inexpensively assessed) is related to worse short-term prognosis. 
They found that mean age rose with increased neutrophil response. After multivariate regression analysis independent predictors were age $>$ or $=75$ years, incomplete revascularization and being in G3. Our study observed that patients with acute ST elevated myocardial infarction who had elevated neutrophil counts had the worse short term prognosis $\mathrm{P}<.001$.

Ghaffari et al. ${ }^{(23)}$ had an agreement with our results. They found that In-hospital mortality and post-STEMI complication rate were $3.7 \%$ and $43.6 \%$, respectively. Higher age $(\mathrm{P}=0.04)$, female gender $(0.002)$, lower ejection fraction $(\mathrm{P}<0.001)$ and absolute neutrophil count $(\mathrm{P}=0.04)$ were predictors of mortality. Pump failure in the form of acute pulmonary edema or cardiogenic shock occurred in $35(8.9 \%)$ of patients. Higher leukocyte $(\mathrm{P}<0.03)$ and neutrophil counts $(\mathrm{P}<0.03)$ and higher NLR $(\mathrm{P}=0.01)$ were predictors of failure. The frequency of ventricular tachyarrhythmias (VT/VF) at the first day was associated with higher neutrophil count $(\mathrm{P}<0.001)$ and higher NLR level $(\mathrm{P}<0.001)$.

\section{CONCLUSION}

The major findings of the present study are:

- Patients with STEMI associated with neutrophilia are significantly associated with early development of congestive heart failure

- Patients with STEMI associated with neutrophilia may help in the identification of individuals at high risk who might benefit from more aggressive interventions to prevent or reduce the risk of CHF

- There is direct positive correlation between leukocyte counts and myocardial infarct size and LV function determined by echocardiography

- Our findings support previous reports demonstrating the prognostic importance of the neutrophil count for coronary events.

- Higher neutrophil count is found to have the best predictive value for both mortality and heart failure.

\section{RECOMMENDATIONS}

- The association between neutrophilia on admission in patients with STEMI and the early development of CHF may help in the identification of individuals at high risk who might benefit from more aggressive interventions to prevent or reduce the risk of $\mathrm{CHF}$
- Our findings demonstrated the prognostic importance of the neutrophil count for coronary events.

- If the peripheral neutrophil count truly reflects the myocardial inflammatory response, future interventions that are designed to limit this response could help to reduce morbidity and mortality rates associated with CHF occurring after STEMI.

\section{REFERENCES}

1. Bursi F, Weston SA, Killian JM et al. (2007): Reactive protein and heart failure after myocardial infarction in the community. Am J Med., 120:616-622.

2. Bhatt DL, Chew DP, Lincoff AM et al. (2003): Effect of revascularization on mortality associated with an elevated white blood cell count in acute coronary syndromes. Am J Cardiol., 92:136-140.

3. Naruko T, Ueda M, Haze K et al. (2002): Neutrophil infiltration of culprit lesions in acute coronary syndromes. Circulation, 106: 2894-900.

4. Zhao G, Wang S, Wang $Z$ et al. (2013): CXCR6 deficiency ameliorated myocardial ischemia/ reperfusion injury by inhibiting infiltration of monocytes and IFN-gammadependent autophagy. Int J Cardiol., 168:853-62.

5. Lorraine $\mathrm{K}$, Jeffery $\mathrm{M}$, Eric $\mathrm{K}$ et al. (2000): Neutrophilia and congestive heart failure after acute myocardial infarction. American heart Journal, 139, 94-100.

6. Cannon CP, McCabe CH, Wilcox RG et al. (2001): Association of white blood cell count with increased mortality in acute myocardial infarction and unstable angina pectoris. OPUS-TIMI 16 Investigators. Am J Cardiol.,87:636-639

7. Barron HV, Cannon CP, Murphy SA et al. (2000): Association between white blood cell count, epicardial blood flow, myocardial perfusion, and clinical outcomes in the setting of acute myocardial infarction: a thrombolysis in myocardial infarction. Circulation, 102:2329-2334.

8. Hoffman R, Benz EJ, Silberstein LE et al. (2013): Hematology: Basic Principles and Practice. 6th ed. Philadelphia, Pa.: Elsevier/Saunders; PP. 164-20.

9. Chobanian AV, Bakris GL, Black HR et al. (2003): National Heart, Lung, and Blood Institute Joint National Committee on Prevention, Detection, Evaluation, and 
Treatment of High Blood Pressure. JAMA., 289 (19):2560-72.

10. Cowie CC, Rust KF, Byrd-Holt DD et al. (2010): Prevalence of diabetes and high risk for diabetes using A1C criteria in the U.S. population in 1988- 2006. Diabetes Care, 33(3):562- 8.

11. Grundy SM, Pasternak R, Greenland P et al. (1999): AHA/ACC scientific statement: Assessment of cardiovascular risk by use of multiple-risk-factor assessment equations. J Am Coll Cardiol., 34(4): 1348-59.

12. Chia S1, Nagurney JT, Brown DF et al. (2009): Association of Leukocyte and Neutrophil Counts With Infarct Size, Left Ventricular Function and Outcomes After Percutaneous Coronary Intervention for STElevation Myocardial Infarction Am J Cardiol ., 103(3):333-7.

13. Lorraine $P$, KyneMB, MP Ha et al. (2000): Neutrophilia and congestive heart failure after acute myocardial infarction. Am Heart J., 139:94-100.

14. Börekçi A, Gür M, Türkoğlu $\mathrm{C}$ et al. (2016): Neutrophil to Lymphocyte Ratio Predicts Left Ventricular Remodeling in Patients with ST Elevation Myocardial Infarction after Primary Percutaneous Coronary Intervention. Korean Circ J., 46(1):15-22.

15. Klein A, Wiberg S, Hassager $\mathrm{C}$ et al. (2019): Admission Leukocyte Count is Associated with Late Cardiogenic Shock Development and All-Cause 30-Day Mortality in Patients with St-Elevation Myocardial Infarction. https://www.ncbi.nlm.nih.gov/pubmed/310 58719

16. Oncel RC, Ucar M, Karakas MS et al. (2015): Relation of neutrophil-tolymphocyte ratio with GRACE risk score to in-hospital cardiac events in patients with
ST-segment elevated myocardial infarction. Clin Appl Thromb Hemost., 21(4):383-8.

17. Tanriverdi Z, Colluoglu T, Dursun $H$ et al. (2017): The Relationship between neutrophil-to-lymphocyte ratio and fragmented QRS in acute STEMI patients treated with primary PCI. J Electrocardiol., 50(6):876-883.

18. Arbel Y, Shacham Y, Ziv-Baran T et al. (2014): Higher neutrophil/ lymphocyte ratio is related to lower ejection fraction and higher long-term all-cause mortality in STelevation myocardial infarction patients. Can J Cardiol., 30(10):1177-82.

19. Yalcinkaya E, Yuksel UC, Celik M et al. (2015): Relationship between neutrophil-tolymphocyte ratio and electrocardiographic ischemia grade in STEMI. Arq Bras Cardiol., 104(2):112-9.

20. Gul U, Kayani AM, Munir R et al. (2017): Neutrophil Lymphocyte Ratio: A Prognostic Marker in Acute ST Elevation Myocardial Infarction. J Coll Physicians Surg Pak., 27(1):4-7.

21. Kirtane AJ, Bui A, Murphy SA et al. (2004): Association of peripheral neutrophilia with adverse angiographic outcomes in ST-elevation myocardial infarction. Am J Cardiol., 93(5):532-6.

22. Fiarresga AJ, Ferreira RC, Feliciano J et al. (2004): Prognostic value of neutrophil response in the era of acute myocardial infarction mechanical reperfusion. Rev Port Cardiol ., 23(11):1387-96.

23. Ghaffari S, Nadiri M, Pourafkari L et al. (2014): The predictive Value of Total Neutrophil Count and Neutrophil/Lymphocyte Ratio in Predicting In-hospital Mortality and Complications after STEMI. J Cardiovasc Thorac Res., 6(1):35-41. 